\title{
BMJ Open Study protocol for the systematic review and meta-analyses of the association between schizophrenia and bone fragility
}

\author{
Behnaz Azimi Manavi (D) ,' Amanda L Stuart (D) , ${ }^{1}$ Julie A Pasco (D) , ${ }^{1,2}$ \\ Jason M Hodge, ${ }^{1,3}$ Kayla Corney (D) , ${ }^{1}$ Michael Berk (D) , ${ }^{1,4,5,6,7}$ Lana J Williams (D) ${ }^{1}$
}

To cite: Azimi Manavi B, Stuart AL, Pasco JA, et al. Study protocol for the systematic review and metaanalyses of the association between schizophrenia and bone fragility. BMJ Open 2020;10:e041859. doi:10.1136/ bmjopen-2020-041859

- Prepublication history for this paper is available online. To view these files, please visit the journal online (http://dx.doi. org/10.1136/bmjopen-2020041859).

Received 20 June 2020

Revised 10 November 2020

Accepted 21 November 2020

Check for updates

(C) Author(s) (or their employer(s)) 2020. Re-use permitted under CC BY-NC. No commercial re-use. See rights and permissions. Published by BMJ.

For numbered affiliations see end of article.

Correspondence to Ms Behnaz Azimi Manavi; bazimimanavi@deakin.edu.au

\section{ABSTRACT}

Introduction Individuals with schizophrenia are known to be at higher risk of comorbid conditions, both physical and psychological. Osteoporosis is possibly one of these, leading to public health concerns due to higher rates of associated mortality and morbidity. We aim to systematically search all available evidence across electronic databases regarding the relationship between schizophrenia and bone fragility.

Methods and analysis A systematic search of the research databases CINAHL, MEDLINE Complete, Embase and PsycINF0 will be conducted and identified papers reviewed for eligibility, with a second reviewer confirming inclusions. Searches will be run from database inception to 1 October 2020 and supplemented by the hand checking of references of identified articles. A previously published scoring system will be used for assessing the methodological quality and risk of bias. A meta-analysis is planned.

Ethics and dissemination Due to including published literature only, ethical permission will not be necessary. Results of this study will be published in a relevant scientific journal and presented at a conference in the field of interest.

PROSPERO registration number CRD42020171959.

\section{INTRODUCTION}

Schizophrenia is a severe and chronic relapsing disorder associated with marked functional impairment. ${ }^{1}$ The lifetime prevalence of schizophrenia is approximately $1 \%$, with the incidence nearing 1.5 per 10000 people. ${ }^{2}$ In Australia, the number of patients experiencing psychosis and receiving treatment in a period of 1 month is about 4.7 per $1000 .{ }^{3}$ This disease is prevalent in both males and females, although symptoms generally develop earlier in men. ${ }^{4}$ Schizophrenia has been attributed to an increased risk of a number of health conditions across various systems, including metabolic syndrome, cardiovascular disease, diabetes, ${ }^{56}$ obstetric complications and cognitive impairments compared with the general population. ${ }^{78}$ Osteoporosis, or bone fragility, is

\section{Strengths and limitations of this study}

- We will apply comprehensive literature searches including index terms, entry terms and keywords.

- Two independent reviewers will extract the data and assess the methodological integrity of each study.

- Studies will not be excluded based on language or nationality of the studied populations.

- The planned meta-analysis is contingent on quantity, quality and/or heterogeneity of available evidence.

- There is a possibility that indigenous populations may not be captured.

another condition that has more recently come under the spotlight.

Osteoporosis is 'a systemic skeletal disease characterised by low bone density and microarchitectural deterioration of bone tissue, with a consequent increase in bone fragility and susceptibility to fracture'. ${ }^{9}$ Due to the higher rates of mortality, morbidity and disability stemming from osteoporosis, it is of significant public health concern. ${ }^{10}{ }^{11}$ In 2011, it was estimated that more than 1.2 million Australians had osteoporosis, ${ }^{12}$ with this expected to reach 6.2 million by $2022 .{ }^{13}$ Tatangelo et al reported the direct annual cost of osteoporosis, osteopenia (low bone mass) and fracture for those aged 50 or older was $\$$ A3.44 billion. ${ }^{14}$

Approximately 20 years ago, the high incidence of osteoporosis and osteoporotic fractures in patients with schizophrenia was first noted. ${ }^{15} 16$ Since then, several studies have shown that compared with the general population, people living with schizophrenia have low Bone Mineral Density (BMD) and are at increased risk of fracture and osteoporosis. ${ }^{17-20} \mathrm{~A}$ metaanalysis of the prevalence of low bone mass in individuals with schizophrenia reported that approximately one in eight patients with schizophrenia had osteoporosis, and this disease is over two and a half times more common in people with 
schizophrenia than controls. ${ }^{21}$ In a systematic review of clinical studies comparing BMD in individuals with schizophrenia compared with controls found 15 out of the 16 studies included reported an increased prevalence of osteoporosis among those with schizophrenia. ${ }^{20}$ Other bone endpoints in the context of schizophrenia including bone quality, bone loss over time and bone turnover are yet to be investigated systematically.

The cause of the observed deficits in BMD in these patients is complex and likely to be multifactorial. $^{2122}$ Both the disease ${ }^{23}$ and related lifestyle/ medical factors ${ }^{24}$ associated with schizophrenia itself are likely to all play a role (eg, smoking, ${ }^{25} 26$ alcohol abuse,${ }^{22}{ }^{27}$ sedentary lifestyle, ${ }^{25}$ reduced exposure to sunlight, ${ }^{28}$ vitamin $\mathrm{D}^{29}$ and calcium deficiency, poor nutrition, ${ }^{30} 31$ diabetes mellitus ${ }^{32}$ and polydipsia. ${ }^{33}$ Furthermore, antipsychotic drugs themselves are associated with an increased risk of osteoporosis and fracture, compounding this association. ${ }^{34} 35$

\section{Objectives}

This aim of this systematic review is to:

1. Identify studies investigating an association between schizophrenia and bone fragility (defined as BMD, bone loss, osteoporosis, fracture, bone quality and bone turnover).

2. Assess the quality of each included study.

3. Identify any potential confounding and/or mediating factors in the link between schizophrenia and bone fragility.

\section{METHODS}

\section{Eligibility criteria}

Cross-sectional, case-control and/or cohort studies investigating the association between schizophrenia (defined by medical records or diagnoses based on Diagnostic and Statistical Manual of Mental Disorders or International Classification of Disease criteria) and bone fragility (defined as BMD, bone loss, osteoporosis, fracture, bone quality and bone turnover) in samples of adults aged $\geq 18$ years, of any nationality and published in any year or language are eligible for inclusion. Clinical trials, grey literature, case reports, theses and conference presentations are ineligible.

\section{Search strategy}

Studies will be identified via electronic searches of research databases in the area of medical, health and social sciences (CINAHL Complete, Embase, MEDLINE Complete and PsycINFO). Searches will be conducted up to 1 October 2020. The following index terms (CINAHL SH/Emtree/MeSH/APA Thesaurus PIT) will be searched: "schizophrenia" AND ("osteoporosis" OR "bone disease, metabolic" OR "fractures, bone" OR "bone and bones" OR "bone density" OR "absorptiometry, photon"). The entry terms of each $\mathrm{MeSH}$ will be searched as title and abstract (TI/ $\mathrm{AB})$. The entry terms for "absorptiometry, photon" are "Dual energy x-ray absorptiometry, DXA, DEXA, densitometry". The entry terms for "bone diseases, metabolic" are "osteopenia, bone loss". The entry terms for "bone density" are "bone mineral density, BMD". The following keywords will also be included: quantitative heel ultrasound, bone turnover markers, bone health, bone fragility and bone quality. Relevant truncation and wildcard symbols will be applied for each database if appropriate.

\section{Data management and extraction}

The online reference management database, Covidence, ${ }^{36}$ will be used for data management. Citation screening and full-text review, finding and removing of duplicated references and extraction of study characteristics and outcomes will be undertaken in this programme. The search strategy will be undertaken by the first reviewer to identify eligible articles. The first reviewer will also handsearch reference lists of the included studies. A further reviewer will confirm the eligibility of the identified articles. Translators will be used if articles are identified in languages other than English.

\section{Assessment of methodological quality}

Methodological quality will be determined using the scoring system by Lievense $e t$ al. ${ }^{37}$ Two reviewers will independently score included studies, with a third providing final judgement should any discrepancy in scores arise. A meta-analysis is planned, however, if not possible due to methodological heterogeneity, a 'best evidence synthesis will be undertaken.

\section{Patient and public involvement}

There was no patient involvement.

\section{Presenting and reporting results}

Preferred Reporting Items for Systematic Reviews and Meta-Analyses (PRISMA) Protocol guidelines ${ }^{38}$ have been followed and the review will conform to PRISMA reporting guidelines. ${ }^{39}$ The Quality of Reporting of Meta-analyses (QUOROM) diagram will be used to document numbers and reasons concerning included vs excluded studies in the context of the prespecified eligibility criteria. ${ }^{40}$

Factors playing a role in the association between schizophrenia and bone fragility will be identified. These factors may consist of related lifestyle/medical factors, such as smoking, alcohol abuse, sedentary lifestyle, vitamin D and calcium deficiency, poor nutrition, diabetes mellitus and polydipsia.

We intend to conduct a meta-analysis; nevertheless, a 'best evidence synthesis ${ }^{41}$ will be completed if a numeral synthesis is not achievable due to methodological heterogeneity. The level of evidence will be categorised using 
four categories ranging from no evidence to strong evidence.

\section{Ethics and Dissemination}

Results will be presented in a related scientific journal and findings presented at scientific conference/s relevant to mental healthand bone.

Due to including published data only, ethical permission is not required. Nevertheless, ethical and governance standards will be abided by, in respect to data management, presentation and dissemination of results.

\section{DISCUSSION}

This systematic review will identify andevaluate the currently available evidence regarding the association between schizophrenia and bone fragility. Furthermore, this review will provide an up-to-date evidence base for which public health strategies aimed at reducing the burden associated with bone fragility associated with schizophrenia could be founded.

\section{Author affiliations}

${ }^{1}$ Deakin University, IMPACT - Institute for Mental and Physical Health and Clinical Translation, School of Medicine, Barwon Health, Geelong, Victoria, Australia ${ }^{2}$ Clinical and Biomedical Sciences: Barwon Health, University of Melbourne School of BioSciences, Melbourne, Victoria, Australia

${ }^{3}$ Geelong Centre for Emerging Infectious Diseases (GCEID), Barwon Health, Geelong, Victoria, Australia

${ }^{4}$ University of Melbourne, Department of Psychiatry, Royal Melbourne Hospital, Parkville, Victoria, Australia

${ }^{5}$ Florey Institute for Neuroscience and Mental Health, University of Melbourne, RoyalMelbourne Hospital, Parkville, Victoria, Australia

${ }^{6}$ Centre of Youth Mental Health, University of Melbourne, Parkville, Victoria, Australia ${ }^{7}$ Orygen, The National Centre of Excellence in Youth Mental Health, Parkville, Victoria, Australia

Acknowledgements The authors want to acknowledge the help and support of Blair Kelly, Librarian, Deakin University and Dr Mohammadreza Mohebbi, Biostatistician, Deakin University.

Contributors BAM, JAP and LJW conceptualised the research question for this protocol. ALS, JMH, KC and MB revised and edited the research question. The search strategy was developed by BAM, JAP and LJW and reviewed by a librarian (BK). The methodological processes have been revised and approved by all authors (BAM, ALS, JAP, JMH, KC, MB and LJW). BAM and LJW drafted this manuscript. All authors (BAM, ALS, JAP, JMH, KC, MB and LJW) read, edited and approved the final version and guarantee the review.

Funding The study is supported by the National Health and Medical Research Council (NHMRC), Australia (1162867). BAM is supported by Deakin University Postgraduate Research Scholarship (DUPRS), KC is supported by an Australian Rotary Health/Bing Taylor PhD Scholarship, MB is supported by an NHMRC Senior Principal Research Fellowship (1156072) and LJW is supported by an NHMRC Career Development Fellowship (1064272) and an NHMRC Investigator grant (1174060).

\section{Competing interests None declared.}

Patient and public involvement Patients and/or the public were not involved in the design, or conduct, or reporting, or dissemination plans of this research.

Patient consent for publication Not required.

Provenance and peer review Not commissioned; externally peer reviewed.

Open access This is an open access article distributed in accordance with the Creative Commons Attribution Non Commercial (CC BY-NC 4.0) license, which permits others to distribute, remix, adapt, build upon this work non-commercially, and license their derivative works on different terms, provided the original work is properly cited, appropriate credit is given, any changes made indicated, and the use is non-commercial. See: http://creativecommons.org/licenses/by-nc/4.0/.

\section{ORCID iDs}

Behnaz Azimi Manavi http://orcid.org/0000-0001-7450-1877

Amanda L Stuart http://orcid.org/0000-0001-8770-9511

Julie A Pasco http://orcid.org/0000-0002-8968-4714

Kayla Corney http://orcid.org/0000-0003-4789-0007

Michael Berk http://orcid.org/0000-0002-5554-6946

Lana J Williams http://orcid.org/0000-0002-1377-1272

\section{REFERENCES}

1 Kane JM, Correll CU. Past and present progress in the pharmacologic treatment of schizophrenia. J Clin Psychiatry 2010;71:1115-24.

2 McGrath J, Saha S, Chant D, et al. Schizophrenia: a Concise overview of incidence, prevalence, and mortality. Epidemiol Rev 2008;30:67-76.

3 Jablensky A, McGrath J, Herrman $\mathrm{H}$, et al. People living with psychotic illness: an Australian study 1997-98: overview of the methods and results of the study of low prevalence (psychotic) disorders as part of the National survey of mental health and wellbeing. Canberra, ACT: Mental Health Branch, Department of Health and Aged Care, 1999.

4 Häfner H. Gender differences in schizophrenia. Psychoneuroendocrinology 2003;28:17-54.

5 Vancampfort D, Correll CU, Galling B, et al. Diabetes mellitus in people with schizophrenia, bipolar disorder and major depressive disorder: a systematic review and large scale meta-analysis. World Psychiatry 2016;15:166-74.

6 Vancampfort D, Stubbs B, Mitchell AJ, et al. Risk of metabolic syndrome and its components in people with schizophrenia and related psychotic disorders, bipolar disorder and major depressive disorder: a systematic review and meta-analysis. World Psychiatry 2015;14:339-47.

7 Chang S-C, Lu M-L. Metabolic and cardiovascular adverse effects associated with treatment with antipsychotic drugs. Journal of Experimental \& Clinical Medicine 2012;4:103-7.

8 Koch E, Rosenthal B, Lundquist A, et al. Interactome overlap between schizophrenia and cognition. Schizophr Res 2020;222:167-74.

9 Simon JA, Mack CJ. Prevention and management of osteoporosis. Clin Cornerstone 2003;5:S5-12.

10 Kanis JA. Diagnosis of osteoporosis and assessment of fracture risk. Lancet2002 1929;359.

11 Kanis JA, Cooper C, Rizzoli R, et al. European guidance for the diagnosis and management of osteoporosis in postmenopausal women. Osteoporosis International 2019;30:3-44.

12 Sugawara N, Yasui-Furukori N, Fujii A, et al. No association between bone mass and prolactin levels among patients with schizophrenia. Hum Psychopharmacol 2011;26:596-601.

13 Watts JJ. Abimanyi-Ochom J, Sanders, Km. osteoporosis costing all Australians a new burden of disease analysis - 2012 to 2022. Osteoporosis Australia 2013 http://hdl.handle.net/10536/DRO/DU: 30060270

14 Tatangelo G, Watts J, Lim K, et al. The cost of osteoporosis, osteopenia, and associated fractures in Australia in 2017. J Bone Miner Res 2019;34:616-25.

15 Abraham G, Friedman RH, Verghese C, et al. Osteoporosis and schizophrenia: can we limit known risk factors? Biol Psychiatry 1995;38:131-2.

16 Delva NJ, Crammer JL, Jarzylo SV, Lawson JS, Owen JA, et al. Osteopenia, pathological fractures, and increased urinary calcium excretion in schizophrenic patients with polydipsia. Biol Psychiatry 1989;26:781-93.

17 Stubbs B, Gaughran F, Mitchell AJ, et al. Schizophrenia and the risk of fractures: a systematic review and comparative meta-analysis. Gen Hosp Psychiatry 2015;37:126-33.

18 Tsai K-Y, Lee C-C, Chou Y-M, et al. The risks of major osteoporotic fractures in patients with schizophrenia: a population-based 10-year follow-up study. Schizophr Res 2014;159:322-8.

19 Wu H, Deng L, Zhao L, et al. Osteoporosis associated with antipsychotic treatment in schizophrenia. Int J Endocrinol 2013;2013:1-7.

20 Kishimoto T, De Hert M, Carlson HE, et al. Osteoporosis and fracture risk in people with schizophrenia. Curr Opin Psychiatry 2012;25:415-29.

21 Stubbs B, De Hert M, Sepehry AA, et al. A meta-analysis of prevalence estimates and moderators of low bone mass in people with schizophrenia. Acta Psychiatr Scand 2014;130:470-86. 
22 Okita K, Kanahara N, Nishimura M, et al. Second-Generation antipsychotics and bone turnover in schizophrenia. Schizophr Res 2014;157:137-41.

23 Partti K, Heliövaara M, Impivaara O, et al. Skeletal status in psychotic disorders: a population-based study. Psychosom Med 2010;72:933-40.

24 Lean M, De Smedt G. Schizophrenia and osteoporosis. Int Clin Psychopharmacol 2004;19:31-5.

25 Cengiz A, Altınyazar V, Manoğlu B, et al. Bone mineral density in patients treated with antipsychotics. Anatolian Journal of Psychiatry 2019;20:182-8.

26 Jung DU, Kelly DL, Oh M-K, et al. Bone mineral density and osteoporosis risk in older patients with schizophrenia. J Clin Psychopharmacol 2011;31:406-10.

27 Troy LH, Elizabeth B-C. A prospective study of alcohol consumption and bone mineral density. BMJ: British Medical Journal 1993;306:1506.

28 Halbreich U, Palter S. Accelerated osteoporosis in psychiatric patients: possible pathophysiological processes. Schizophr Bull 1996;22:447-54.

29 Pasco JA, Henry MJ, Nicholson GC, et al. Behavioural and physical characteristics associated with vitamin D status in women. Bone 2009;44:1085-91.

30 Peet M. Diet, diabetes and schizophrenia: review and hypothesis. $\mathrm{Br}$ J Psychiatry Suppl 2004;47:s102-5.

31 Wark JD. Osteoporotic fractures: background and prevention strategies. Maturitas 1996;23:193-207.

32 Holloway-Kew KL, Marijanovic N, De Abreu LF, et al. Bone mineral density in diabetes and impaired fasting glucose. Osteoporosis International 2019;30:1799-806.
33 Misra M, Papakostas GI, Klibanski A. Effects of psychiatric disorders and psychotropic medications on prolactin and bone metabolism. $J$ Clin Psychiatry2004 1590;65::1607-1618;-1760. quiz.

34 Naidoo U, Goff DC, Klibanski A. Hyperprolactinemia and bone mineral density: the potential impact of antipsychotic agents. Psychoneuroendocrinology 2003;28:97-108.

35 Lee SH, Hsu WT, Lai CC, et al. Use of antipsychotics increases the risk of fracture: a systematic review and meta-analysis. Osteoporos Int 2017;28:1167-78.

36 Covidence Systematic Review Software, Veritas Health Innovation: Melbourne, Australia; [updated; cited]. Available from: www. covidence. org.

37 Lievense A, Bierma-Zeinstra S, Verhagen A, et al. Influence of work on the development of osteoarthritis of the hip: a systematic review. Journal of Rheumatology 2001;28:2520-8.

38 Moher D, Shamseer L, Clarke M, et al. Preferred reporting items for systematic review and meta-analysis protocols (PRISMA-P) 2015: elaboration and explanation. 4. BMJ: British Medical Journal, 2015.

39 Moher D, Liberati A, Tetzlaff J, et al. Preferred reporting items for systematic reviews and meta-analyses: the PRISMA statement. BMJ 2009;339:b2535-336.

40 Brennan SL, Pasco JA, Urquhart DM, et al. The association between urban or rural locality and hip fracture in community-based adults: a systematic review. Journal of Epidemiology \& Community Health 2010;64:656-65.

41 Chandrasekaran V, Brennan-Olsen SL, Stuart AL, et al. Association between bipolar spectrum disorder and bone health: a meta-analysis and systematic review protocol. BMJ Open 2017;7:e013981. 